\title{
LBM Analysis of Micro-Convection in MHD Nanofluid Flow
}

\author{
Kozhikkatil Sunil Arjun* - Rakesh Kumar \\ Department of Mechanical Engineering, Indian Institute of Technology (ISM) Dhanbad, India
}

The lattice Boltzmann-Bhatnagar-Gross-Krook method was used to simulate $\mathrm{Al}_{2} \mathrm{O}_{3}$-water nanofluid to find the effects of Reynolds, Rayleigh and Hartmann numbers, slip coefficient, nanoparticle volume fraction and axial distance on forced convection heat transfer in MATLAB. The ranges of studied Reynolds number, Rayleigh number, magnetic field strength, nanoparticle volume concentration and slip coefficient include $200 \leq R e \leq 4000 ; 103 \leq R a \leq 106 ; 0 \leq \mathrm{Ha} 90 ; 0 \leq \varphi \leq 2 \% ; 0.005 \leq B \leq 0.02$, respectively. The results show that increasing Reynolds number and nanoparticle volume fractions improve heat transfer in the 2D microtube under laminar, turbulent, slip and temperature jump boundary conditions. Decreasing the values of slip coefficient decreases the temperature jump and enhances the Nusselt number. A critical value for the Rayleigh number (105) and magnetic field strength (Ha 10) exists, at which the impacts of the solid volume fraction and slip coefficient effects are the most pronounced. The pressure drop shows a similar type of enhancement in magnitude, as observed in the case of the Nusselt number. However, application of nanofluids for low Reynolds numbers is more beneficial, and the effect of volume fractions are more pronounced in comparison to slip coefficient, though the effects are marginal.

Keywords: magneto-hydrodynamics, Nusselt number, lattice Boltzmann method, microtube, slip coefficient

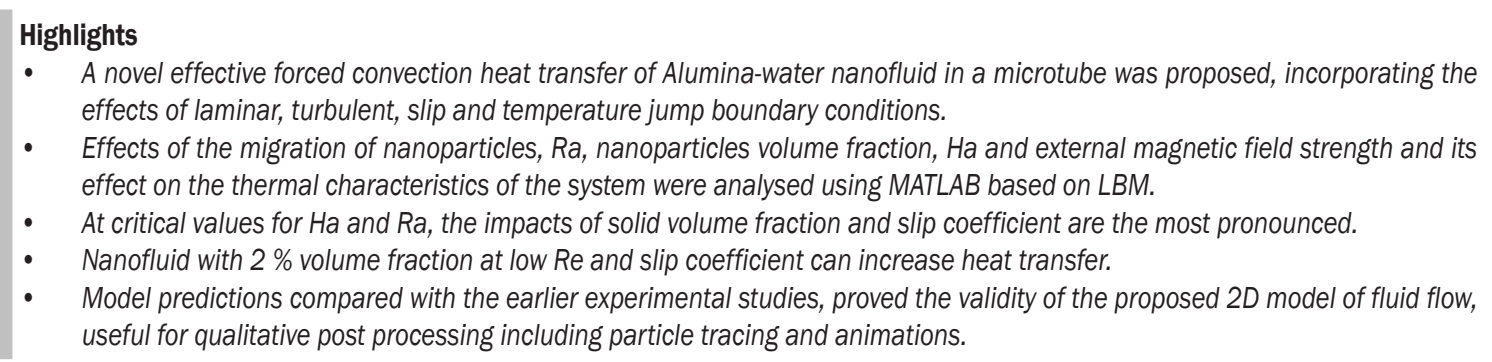

\section{O INTRODUCTION}

The external magnetic field can suppress or enhance the heat transfer of nanofluid flowing through a micro-channel by adjusting its orientation and magnitude. CFD simulate flow conditions that are not reproducible during experimental tests that are too large, remote, or small. Considering the difficulties of experimental techniques to determine extremely low permeability, the lattice Boltzmann method is easier to implement, computationally efficient over conventional computational fluid dynamics (CFD) and more capable for the simulation of micro, slip and transition regimes, and large complex flows and interactions between different phases, based on its intrinsically mesoscopic kinetic nature. Expensive computation and complex mathematical procedure of molecular dynamics and direct simulation Monte Carlo, as well as the inability of Navier-Stokes for simulation of flow in transition and free molecular regimes, have encouraged the use of lattice Boltzmann methods (LBM) [1]. It is a finite difference method, and the Navier-Stokes equations can be recovered with a proper choice of the collision operator.
The information theory with measures to quantify information contents is based on the notion of causal states as stochastic spatio-temporal patterns capturing the dynamics of a local neighbourhood and applicable to structured time-dependent spatial data. Causal states that feature unusual behaviour over time intervals can then be quantified. When flows become more complex, 2D hydrodynamic modelling provides a better indication of extents and characteristics. 3D models are difficult to create and more difficult to read. 2D model distributes the fluid more effectively throughout the area and has the ability to allow for variance within the $X Y$ plane of elevation and material roughness. When it is anticipated that there will be a significant interaction between flow paths or breakout from channels, a 2D hydrodynamic model will provide a more comprehensive indication of characteristics throughout the study area.

Magnetorheological fluid film bearings are evaluated for an SAE-10 W lubricant with controllable properties and magnetic particles aligned into chains, capable of excitement as Magnetorheological fluid, in which a magnetostatic field affects the apparent viscosity [2]. Thermal conductivity enhancement and thermomagnetic convection in devices using magnetic 
nanofluids are capable of controlling its flow and heat transfer process via an external magnetic field [3]. The noncovalent surface coating of CNT's with magnetite nanoparticles composite based magnetorheological fluid possesses an increased sedimentation stability, larger saturation critical stresses, and faster response to time- varying magnetic fields better than ferrofluids and conventional magnetorheological fluids do [4]. The heat transfer of a heat pipe filled with a ferrofluid, stabilized by citrate ions, was enhanced by about $13 \%$ [5]. The heat transfer in the electrorheological fluids considering the microconvection transport is numerically solved on a flat layer, and it is concluded that the internal rotations of elementary particles intensify heat transfer [6]. The increase in particle volume fraction and magnetic field strength may increase the thermal conductivity of the magnetorheological fluid, a smart material with the capability of changing reversibly from liquid to near solid state under the presence of external magnetic fields [7]. Nano-sized magnetic particle-based Ferro Fluid is a magnetic colloidal suspension of two different nano-sized magnetic particles, dispersed in a carrier liquid; it has improved shear thinning, elevated dynamic moduli and thermo-rheological complexity due to a long chain-like structure under the influence of an applied magnetic field [8].

The lattice Boltzmann method [9] originated from Ludwig Boltzmann's kinetic theory of gases. Gases/fluids can be imagined as consisting of a large number of small particles with random motions, and the exchange of momentum and energy is achieved through particle streaming and billiard-like particle collision. The number of particles is reduced and confined to the nodes of a lattice in LBM. For a 2D model, known as the $\mathrm{D} 2 \mathrm{Q} 9$, a particle is restricted to stream in nine possible directions, including one staying at rest; these velocities are referred to as the microscopic velocities. For each particle on the lattice, a discrete probability distribution function is associated, which describes the probability of streaming in one specific direction. The macroscopic fluid density is defined as the summation of microscopic particle distribution functions and the macroscopic velocity is an average of microscopic velocities, weighted by the distribution functions.

There is no report of magneto-hydro-dynamics (MHD) mixed convective heat transfer of nanofluids in a vertical microtube, except a theoretical study of fully developed convective heat transfer of nanofluid under a uniform magnetic field using a modified Buongiorno's model [10]. Studies on the convective heat transfer of nanofluids in microducts are limited.
The heat transfer of non-Newtonian nanofluids in a microtube rises with an increase in volume fraction but decreases with an increase in diameter [11]. Theoretical and experimental convective heat transfer analysis of stable magnetic nanofluids in a microtube under an external magnetic field [12] for velocity, temperature, pressure, pressure drop, and flow drag is reported. The well-known differences of microflows from macroscopic ones are slip velocity and temperature jump on the solid-fluid boundaries. For liquid microflows, a slip flow regime can be observed [13] and hence, Navier-Stokes and particle based methods including LBM can be applied. Convective heat transfer characteristics of microscale flows for optimum design of thermal systems operating at low Re are reported [14]. Compressibility is the dominant factor in high-speed microtube flows, and the rarefaction effect is important for low speed flows in microtubes with relatively low Mach number and high Knudsen number [15].

Use of an internal energy distribution function gives stable results [16] as it considers pressure work and viscous heat dissipation [17] and thus the most stable thermal LBM method. Raisi et al. [18] simulated $\mathrm{Cu}$-water nanofluid in a microchannel for both slip and no-slip conditions, ignoring temperature jump effects and applying the classic Navier-Stokes equations. Theoretical results of fluid flow in slip flow regimes or nanofluid flow simulation using LBM in a single or multi-phase mixture model has been reported [19]. Few studies of nanofluid simulation in microchannels using LBM [20] and [21] have ignored slip velocity and temperature jump effects, except in [1]. An accurate understanding of convection heat transfer of nanofluids in a microtube in the slip flow regime and the effect of temperature jump is not yet available in the literature.

Particular attention was given to the effects of temperature jump and slip velocity with different solid volume fractions of nanofluid in the slip flow regime using LBM. We have investigated the effects of migration of nanoparticles and its effect on the thermal characteristics of the system. We have also analysed the effects of governing parameters such as $R a$, nanoparticles volume fraction, $H a$, and external magnetic field strength. 2D model of fluid flow used in this study can be used for qualitative post processing including particle tracing and animations.

\section{PROBLEM AND NUMERICAL DETAILS}

We have used a steel microtube (thickness $50 \mu \mathrm{m}$, length $10 \mathrm{~mm}$ and internal diameter $100 \mu \mathrm{m}$ ) as 
shown in Fig. 1 to study numerically, using the double population LBM-BGK (Bhatnagar-GrossKrook) model. The BGK model is one of the most popular lattice Boltzmann methods for simulating the convection heat transfer. In this, hydrodynamic and thermal parameters of fluid flow are estimated using density momentum $(f)$ and internal energy density $(g)$ distribution functions. A computer simulation has been developed and written using MATLAB software. Most of the existing LBM-BGK models can be viewed as compressible schemes to simulate incompressible fluid flows. The compressible effect might lead to some undesirable errors in numerical simulations. In this paper, an LBM-BGK model without compressible effect is designed for simulating incompressible flows. The incompressible Navier-Stokes equations are recovered from this incompressible model. The results agree well with the analytic solutions and the results of previous studies.

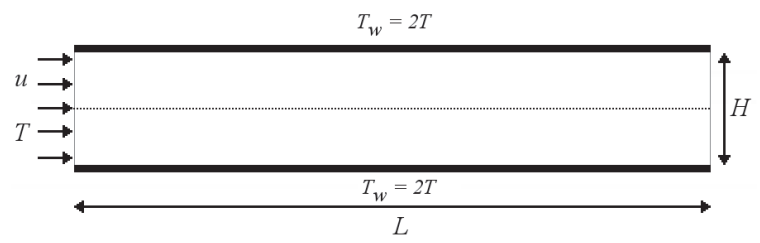

Fig. 1. Geometry of microtube

Let $u$ and $T$ be the velocity and temperature profiles at the inlet. The wall temperature is set to $T_{w}=2 T i$ with the wall surfaces subjected to a uniform heat flux of $5000 \mathrm{~W} / \mathrm{m}^{2}$. As the length of the microtube is long enough, we will obtain fully developed hydrodynamic and thermal conditions rapidly in the turbulent regime, and $R e$ is small. Since the tube is small, tube wall thickness is comparable to the inner diameter, and the heat conduction in the wall along the axis direction may be important. The ratio of axial conduction to the tube inside convection is less than 0.02 and thus can neglect it. The simulated nanofluid is a dispersion of nanoparticles of Alumina with a mean diameter of $23 \mathrm{~nm}$ in pure water. We assume it $\mathrm{pt}$ be an incompressible Newtonian fluid with negligible radiation effect and in laminar and turbulent flow regime. The size of nanoparticles in this study is small enough to be considered as a homogenous flow as reported in the corresponding recent works [22] to [24].

The effect of $\operatorname{Re} 200$ to 4000 with $R a 10^{3}$ to $10^{6}$ under the influence of $H a 0$ to 90 are noted. The effects of the nanoparticle volume fraction $(\varphi=0 \%$ to $2 \%$ ) are investigated for forced convection. The slip velocity, temperature jump, and their effects are studied for different values of slip coefficient, $B=0.005$ to 0.02 along the axial distance 0.25 to the full length of the duct. Here, $\tau$ is the dimensional relaxation time; $f_{i}(x, t)$ is the density distribution function for the particle moving with discrete velocity $C_{i}$ at position $x$ and time $t ; f_{i}^{e}(x, t)$ and $g_{i}^{e}(x, t)$ are the local equilibrium distribution functions that have an appropriately prescribed functional dependence on the local hydrodynamic properties. $C=\left(3 r T_{m}\right)-1 / 2\left(T_{m}\right.$ is the mean value of temperature). $\Delta t$ is the lattice time step which is set to unity, $\tau_{f}$ and $\tau_{g}$ are the relaxation times for the flow and temperature fields, respectively.

The hydrodynamic and thermal Boltzmann equations are written as follows [25] and [26].

$$
\begin{gathered}
\frac{\partial f_{i}}{\partial t}+c_{i \alpha} \frac{\partial f_{i}}{\partial x_{\alpha}}=\Omega(f)=-\frac{1}{\tau_{f}}\left(f_{i}-f_{i}^{e}\right), \\
\frac{\partial g_{i}}{\partial t}+c_{i \alpha} \frac{\partial g_{i}}{\partial x_{a}}=\Omega\left(g_{i}\right)-f_{i} Z_{i}= \\
=0.5|\mathbf{c}-\mathbf{u}|^{2} \Omega\left(f_{i}\right)-f_{i} Z_{i}=-\frac{\left(g_{i}-g_{i}^{e}\right)}{\tau_{g}}-f_{i} Z_{i},
\end{gathered}
$$

where $\mathbf{u}=(u, v)$ and $\Omega$ are the macroscopic velocity vector and collision operator, respectively.

We have applied the D2Q9 lattice model (Fig. 2) to the present study. Microscopic discretized velocities are:

$$
\begin{gathered}
C_{i}=\left(\cos \frac{i-1}{2} \pi, \sin \frac{i-1}{2} \pi\right), \text { when } i=1 \text { to } 4, \\
C_{i}=\sqrt{2}\left(\cos \left[\frac{i-5}{2} \pi+\frac{\pi}{4}\right], \sin \left[\frac{i-5}{2} \pi+\frac{\pi}{4}\right]\right), \\
\text { when } i=5 \text { to } 8, \\
C_{0}=(0,0) .
\end{gathered}
$$

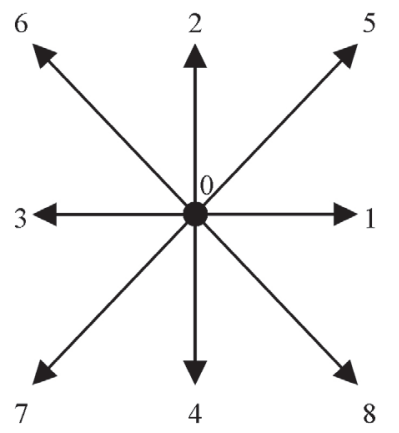

Fig. 2. D2Q9 lattice

The discrete heat dissipation and equilibrium distribution functions are: 


$$
\begin{gathered}
Z_{i}=\left(c_{i \alpha}-u_{\alpha}\right)\left[\frac{\delta u_{\alpha}}{\delta t}+c_{i \alpha} \frac{\partial u_{\alpha}}{\partial x_{\alpha}}\right], \\
f_{i}^{e}=\omega_{i} \rho\left[1+3\left(c_{i} \cdot u\right)+\frac{9\left(c_{i} \cdot u\right)^{2}}{2}-\frac{3 u^{2}}{2}\right], \\
i=0,1, \ldots, 8 ; \quad \omega_{0}=\frac{4}{9} ; \quad \omega_{1,2,3,4}=\frac{1}{9} ; \quad \omega_{5,6,7,8}=\frac{1}{36}, \\
g_{1,2,3,4}^{e}=\frac{1}{9} \rho e\left[\begin{array}{c}
1.5+1.5\left(c_{1,2,3,4} \cdot u\right)+ \\
+4.5\left(c_{1,2,3,4} \cdot u\right)^{2}-1.5 u^{2}
\end{array}\right], \\
g_{5,6,7,8}^{e}=\frac{1}{36} \rho e\left[\begin{array}{c}
3+6\left(c_{5,6,7,8} \cdot u\right)+ \\
+4.5\left(c_{5,6,7,8} \cdot u\right)^{2}-1.5 u^{2}
\end{array}\right] .
\end{gathered}
$$

We have simulated the collision and streaming steps in LBM to overcome difficulty in solving implicit forms as a modified discrete distribution function for density-momentum $\left(\tilde{f}_{i}\right)$,

$$
\begin{gathered}
\tilde{f}_{i}\left(x+c_{i} \Delta t, t+\Delta t\right)-\tilde{f}_{i}(x, t)= \\
=-\frac{\Delta t}{2 \tau_{f}+0.5 \Delta t}\left[\tilde{f}_{i}(x, t)-f_{i}^{e}(x, t)\right], \\
\tilde{f}_{i}=f_{i}+\frac{\Delta t}{2 \tau_{f}}\left(f_{i}-f_{i}^{e}\right),
\end{gathered}
$$

and that for internal energy $\left(\tilde{\mathrm{g}}_{i}\right)$,

$$
\begin{gathered}
\tilde{g}_{i}\left(x+c_{i} \Delta t, t+\Delta t\right)-\tilde{g}_{i}(x, t)= \\
=-\frac{\Delta t}{\tau_{g}+0.5 \Delta t}\left[\tilde{g}_{i}(x, t)-g_{i}^{e}(x, t)\right]-\frac{\tau_{g} \Delta t}{\tau_{g}+0.5 \Delta t} f_{i} z_{i} \\
\tilde{g}_{i}=g_{i}+\frac{\Delta t}{2 \tau_{g}}\left(g_{i}-g_{i}^{e}\right)+\frac{\Delta t}{2} f_{i} z_{i} .
\end{gathered}
$$

The top and bottom boundaries are adiabatic. We have estimated the unknown inlet and outlet thermal distribution functions, satisfying the equilibrium conditions and improving the accuracy using the known inlet temperature profile and non-equilibrium bounce back model, normal to the boundary [27]. We have an applied specular reflective bounce back model (combination of bounce back and specular boundary condition) to determine the slip velocity in LBM, in this work. For example, for the bottom wall, we have estimated the unknown distribution functions by Eqs. (14) and (15). The tangential momentum accommodation coefficient is a parameter in determining the degree of the slip and represents the average tangential momentum exchange between the fluid molecules and the solid boundary. This coefficient is primarily dependent on the roughness of the wall surface and its energetic attraction of fluid molecules; and is near unity for most engineering applications [28]. The accommodation coefficient rises as the wall-fluid attraction strength increases [29]. We have chosen the effect of the energy accommodation coefficient in reducing the slip flow as 0.3 corresponding to the temperature values used in this study to maintain the mass flow rate as per [30]. In addition, after fixing the slip coefficient as 0.2 being the bottom plot as per [30], we have obtained the value of accommodation coefficient $r$, as the sum of the coefficients is unity.

For the different microtubes with various roughnesses, the curves of thermal performance are close to each other. This indicates that although within the 10-mm length range of microtube, the roughness effect on thermal performance exists as an enhancement in nature, flow resistance also increases and the overall thermal performance does not change much. For the same mass flow rate, an increase in roughness will decrease flow area, leading to increased flow velocity and subsequently reduced heat exchange efficiency [31]. The presence of surface roughness reduces boundary slip for flow in microtubes. The decrease in roughness height or increase in Knudsen number can lead to large wall slip for gas flow in microchannels [32]. The microtubes cannot express the comprehensive effects of roughness and boundary slip on MHD nanofluid flow behaviour and heat transfer characteristics.

At low shear rates, the slip length has desired consistency with the Navier model. At high shear rates, the Navier condition fails and boundary condition will be nonlinear even though the liquid remains Newtonian. This phenomenon is just like the Knudsen number rule for linear slip condition of Navier-Stokes equations in dilute gases.

$$
\begin{gathered}
\tilde{f}_{2}=\tilde{f}_{4}, \\
\tilde{f}_{5,6}=r \tilde{f}_{7,8}+(1-r) \tilde{f}_{8,7} .
\end{gathered}
$$

For the D2Q9 model and the horizontal wall boundary with the flow on its upside as shown in Fig. 2, we note that the distribution functions at $\alpha$ is 2,5 and 6 directions, indicating that the flow field from outside the wall are unknown. The distribution functions at $\alpha$ is $1,3,4,7$ and 8 are known because they stream from the points in the flow field. In the diffuse scattering boundary condition, the unknown distribution functions can be determined from the known distribution functions. For the wall boundary as shown in Fig. 2, we have simplified the temperature 
jump for the bottom wall as below in LBM, based on the internal energy distribution function:

$$
\tilde{g}_{2,5,6}=\frac{3}{\rho_{\omega} e} g_{2,5,6}^{e}\left(\rho_{\omega}, u_{\omega}, e_{\omega}\right)\left(\tilde{g}_{4}+\tilde{g}_{7}+\tilde{g}_{8}\right) \text {. }
$$

The dot products of the wall normal vector $\mathbf{n}$ and the lattice velocities at $\alpha$ is 1 and 3 , are zero. We have also calculated the top wall temperature jump similarly.

In previous works in which the temperature jump of nanofluid was not taken into account, $N u=k_{n f} / k_{f}(\partial \theta / \partial Y) w$ with $\theta=(T-T c) /(T h-T c)$. In the present work, the effect of even a small value of temperature jump results in the temperature gradient between the wall and adjacent fluid layer. At this state, $\mathrm{Nu}$ approaches asymptotically a constant value along the microtube walls, the outlet $N u$ at the outlet domain of a microtube.

$$
\begin{gathered}
N u=\left(k_{n f} / k_{f}\right) \frac{D_{H}(\partial T / \partial y)_{w}}{T_{w}-T_{b u l k}}, \\
\operatorname{Re}=\rho_{n f} u_{n f} D_{H} / \mu_{n f} .
\end{gathered}
$$

Calculation of pressure is:

$$
p=\frac{c^{2}}{3} \rho
$$

For natural convection, the Raleigh number is:

$$
\operatorname{Ra}=g \beta L^{3} \operatorname{Pr} / v^{2}, \text { where } \operatorname{Pr}=v / \chi .
$$

In the simulation, Boussinesq approximation applied to the buoyancy force term, as in [33]:

$$
F_{i}=3 \omega_{i} \rho g \beta \Delta T .
$$

The external magnetic field only influences the force term, where new parameter added to the buoyancy force term as:

$$
\begin{gathered}
F_{i}=F_{i x}+F_{i y}, \\
F_{i x}=3 \omega_{i} \rho[A(v \sin \theta \cos \theta-u \sin 2 \theta)], \\
F_{i y}=3 \omega_{i} \rho[g \beta \Delta T+A(u \sin \theta \cos \theta-v \cos 2 \theta)],
\end{gathered}
$$

where $A$ is the adjustable coefficient, $\theta$ is the magnetic field orientation, $\beta$ is the thermal expansion coefficient, and $g$ is the gravitational acceleration. The convergence criterion is that the relative changes of the variables between two successive iterations are less than $10^{-6} \%$.

We have applied the Large Eddy Simulation model in the LBM, with the total viscosity affecting the relaxation time. The effect of the magnetic field was shown only in the force term, which is added to the collision process and investigated for $\mathrm{Ha} 0$ to 90 at $10^{7}<\mathrm{Ra}<10^{9}$ for turbulent flow using $2 \% \mathrm{Al}_{2} \mathrm{O}_{3}$ water nanofluid at $R e 200$.

\section{RESULTS AND DISCUSSION}

The effects of the dimensionless slip coefficient on $\mathrm{Nu}$ along the microtube wall are shown in Figs. 3 to 5 at $R a=10^{4}, R e=200$ and $B=0.005$ to 0.02 for $\varphi$ $=0 \%$ to $2 \%$. Nu has the largest value at the entrance with axial distance 0.25 -microtube length and then starts to decrease and $N u$ increase with $\varphi$. We have observed that the increase of solid concentration leads to the enhancement of heat transfer; such enhancements are due to the increase of the effective thermal conductivity with the increase of solid volume fractions of nanoparticles. Significant values of temperature jump were observed, especially around the entrance region, and have the greatest temperature gradient near the wall.

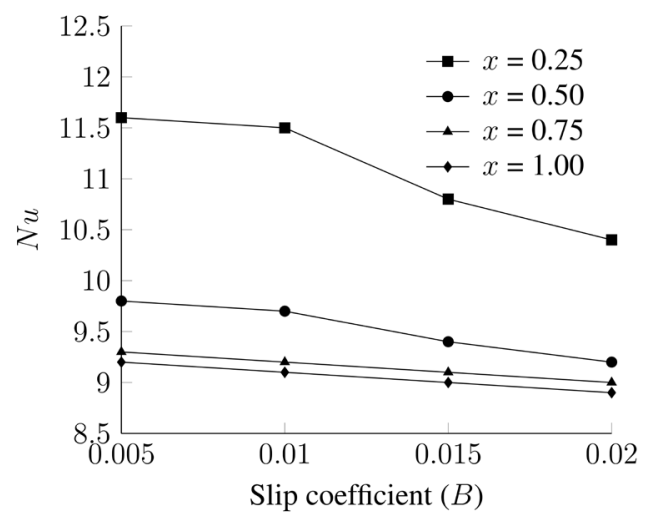

Fig. 3. Effect of slip coefficient on $N u(\varphi=0 \%)$

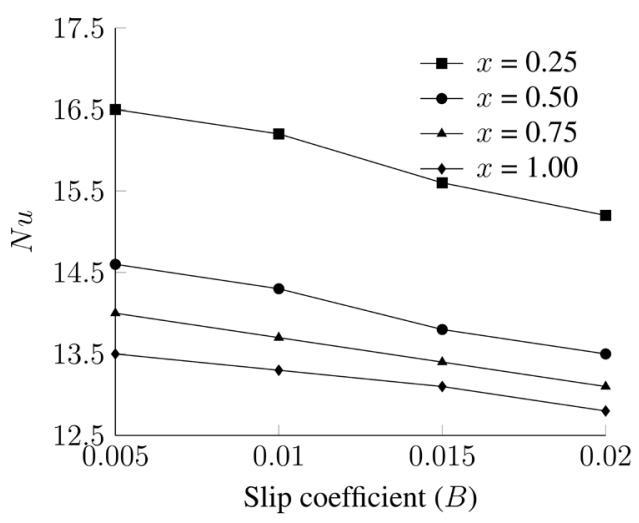

Fig. 4. Effect of slip coefficient on $N u(\varphi=1 \%)$

Observations show that a larger dimensionless slip coefficient corresponds to larger slip velocity as well as temperature jump on the walls, especially at the entrance region. With a larger dimensionless 
slip coefficient, the temperature gradient decreases compared to places where the dimensionless slip coefficient is smaller; consequently, $N u$ will have a lower value. The temperature gradient between the nanofluid particles on the wall and their neighbouring ones adjacent to the wall decreases with a larger dimensionless slip coefficient, and as a result, $\mathrm{Nu}$ would have the lesser value of recent cases. Thus, $\mathrm{Nu}$ results obtained near the walls have higher accuracy if we consider the temperature jump. Further, the decrease in $N u$ is a result of the augmented thermal energy transfer from the wall to the fluid. The nanoparticles hit the wall, absorb thermal energy, reduce the wall temperature, and mix back with the bulk of the fluid. Since the inclusion of nanoparticles enhances the effective thermal conductivity of the nanofluid, the decrease in the $N u$ is more remarkable where the conduction regime prevails $\left(R a 10^{4}\right)$. Thus, the $N u$ decreases by increasing $x$, regardless of the volume concentration.

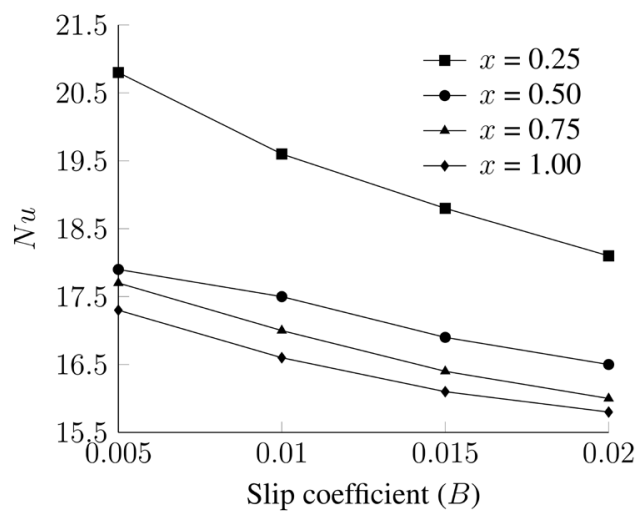

Fig. 5. Effect of slip coefficient on $N u(\varphi=2 \%)$

Most of the previous reports ignored the temperature jump [18], [34] and [35] except [36]. Decreasing the values of slip coefficient increases the wall slip velocity, temperature jump values, convective heat transfer coefficient; consequently, the $\mathrm{Nu}$ at each axial position as well as for all volume fractions of nanofluid. In the present study, thermal enhancement up to $30 \%$ to $32 \%$ could be achieved by decreasing the slip coefficient with the increase of the volume fraction from $0 \%$ to $2 \%$. This finding is in agreement with the results of [36]. In low $R e$ number laminar forced convection flows $(R e=1$ to 10), the Brownian force has a significant effect on flow and heat transfer characteristics, thermophoresis may be neglected, and $N u$ and convection heat transfer coefficient significantly increase [37]. We have captured the maximum $N u$ based on the bulk mean nanofluid thermal conductivity when the ratio of Brownian and thermophoretic diffusivities is 0.5 , which can be useful for designing nanoparticles for high-energy carriers [37].

We have presented $N u$ at the outlet with different values of $\varphi, R a$ at $10^{3}$ to $10^{6}$ and $\operatorname{Re} 200$ in Fig. 6, which indicates the effect of using nanofluid volume fractions and $R a$ to increase the heat transfer rate. When the $R a$ is larger than $10^{5}$, the $N u$ near the heated wall decreases significantly. For a fixed $R a$, solid volume fractions can affect the enhancement of heat transfer. By adding $2 \% \mathrm{Al}_{2} \mathrm{O}_{3}$ nanoparticles by volume, the $N u$ increases about $17 \%$ at $R a=10^{4}$ from $10^{3}$, a slight decline of enhancement to $8 \%$ at $R a=10^{5}$ (but with the highest value of 18.95), but decreases significantly to $33 \%$ when $R a$ increases to $10^{6}$. This means that there is a critical value of $R a$ of $\mathrm{Al}_{2} \mathrm{O}_{3}$-water nanofluid for the performance of heat transfer enhancement in terms of the $\mathrm{Nu}$ and identified as $10^{5}$ in this study.

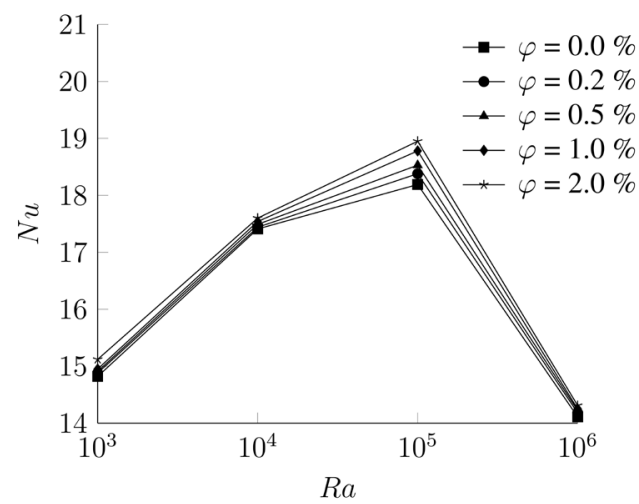

Fig. 6. Effect of nanofluid volume fractions and $\mathrm{Ra}$ on $\mathrm{Nu}$ $(B=0.005)$

We have observed the suppression of the fluid velocity and hydrodynamic boundary layer thickness owing to the retarding effect of increased Lorenz force, by imposing the magnetic field in terms of increasing $\mathrm{Ha}$ at low $\mathrm{Ra}$ from $10^{3}$ to $10^{4}$. The intensity of convection weakens significantly, and the domination of the conduction mode of heat transfer makes the isotherms parallel to each other and maximizes stream function value. The $R a$ compares buoyancy forces to viscous forces. For lower $R a$, viscous forces dominate over buoyancy forces to maintain the conduction regime with small and stable disturbances in the fluid and negligible thermosolutal convection effects on macrosegregation. As conduction is the dominant mechanism alone up to $R a$ $10^{4}, N u$ is minimum compared to that at $R a 10^{5}$. As the 
$R a$ increases up to $10^{5}$, the role of the convection in heat transfer becomes more significant. Consequently, the thermal boundary layer thickness becomes thinner. A rise in velocity gradients leads to a shear stress augmentation at the walls, enhancing the pressure drop. The slip velocity has an increasing trend with the velocity gradient. Thus, momentum closer to the walls enhances and leads to a rise in the convective heat transfer compared to lower $R a$. There is a critical Rayleigh number for heat transfer enhancement of applying nanofluids; beyond the critical value, the enhancement rate will be reduced. At critical $R a$, the thermal diffusivity is high, and heat moves rapidly through it by conduction relative to its volumetric heat capacity. We have obtained the maximum value of $N u$ at $R a 10^{5}$ compared to higher $R a$. The transition from conduction to convection mode of heat transfer change is noticed at $R a 10^{5}$ and saturation happens at this $R a$, which corresponds to critical $R a$. A similar conclusion was obtained in [29].

The mutual effects of the buoyancy and inertia determine flow inside a microtube. These forces run parallel to each other; their directions are opposite for positive values of mixed convective parameters. At $R a$ of $10^{6}$, a similar flow pattern as at $R a 10^{5}$ is observed, but with a greater thermal gradient. The increase in mixed convective parameter intensifies the buoyancy force, which leads to a rise of the momentum near the walls and due to a constant mass flow rate inside the microtube, the velocity in the core region reduces. An increase in mixed convective parameter also reduces the absolute amplitude of the concentration profile in the core region and enhances the concentration near the walls. Above a critical $R a$, buoyancy forces become important, disturbances grow, and a dominant convection regime is established. Here, the effects of thermosolutal convection on macro segregation become significant. The momentum in the core region moves toward the walls, so does the nanoparticle concentration. Hence, the near wall velocity gradients increase with increasing $H a$, enhancing the slip velocity. An upward trend of the velocity at the wall and a reversed behaviour for that in the core region of the microtube, with increasing mixed convective parameter is established. This causes the temperature near the heated wall to decrease significantly. For $R a 10^{6}, \mathrm{Nu}$ decreases in comparison to $R a 10^{5}$. The existence of a critical $R a$ value of $10^{5}$ for the performance of heat transfer enhancement in terms of the normalized average $\mathrm{Nu}$ has been reported [37] and [38] in an enclosure filled with $\mathrm{Al}_{2} \mathrm{O}_{3}$-water nanofluid.

Using $1 \%$ of Alumina nanoparticles leads to an increase of almost $32 \%$ of outlet $N u$ at $R a=10^{5}$.
This increase would be over $34 \%$ for using $4 \%$ of Alumina-water nanofluid. Similar conclusions were already reported [30] and [38]. However, we have observed the effect of volume fractions more pronounced than slip coefficient. Flow behaviours and the average rate of heat transfer in terms of the $\mathrm{Nu}$ as well as the thermal conductivity of nanofluid is effectively changed with the particle volume fraction and particle diameter with a fixed $R a$ [39]. The mean $N u$ increased with increase in $R a$ [40]. The flow and heat transfer characteristics of $\mathrm{Al}_{2} \mathrm{O}_{3}$-water nanofluid in the square cavity are more sensitive to viscosity than to thermal conductivity [41].

The impact of the nanoparticle volume fraction on $N u$ is small when $10^{4}<R a>10^{5}$ and negligible at all other $R a$ values of the investigation (Fig. 6). This indicates that there exist a critical $R a$, at which the impact of nanoparticle volume fraction on $\mathrm{Nu}$ becomes small, and negligible when $R a$ is below and above this critical $R a$. The profiles of $N u_{x}$ for $R a$ values $10^{3}$ to $10^{6}$ along the microtube wall at $R e 200$ and $B=0.005$ for $\varphi=0.02$ is given in Fig. 7. $N u_{x}$ has larger values at the entrance and then start to decrease asymptotically along the walls and approach constant values for all $R a$ values. As $R a$ increases from $10^{3}$ to $10^{4}, N u_{x}$ increases significantly, then at $R a 10^{5}$, the enhancement decreases and at $R a 10^{6}, N u_{x}$ values decreases to a minimum and lower than those at $R a$ $10^{3}$. Hence, $R a 10^{5}$ is the critical $R a$ that determines the impact of nanoparticle volume fraction on $N u$ at a constant $R e$ and dimensionless slip coefficient.

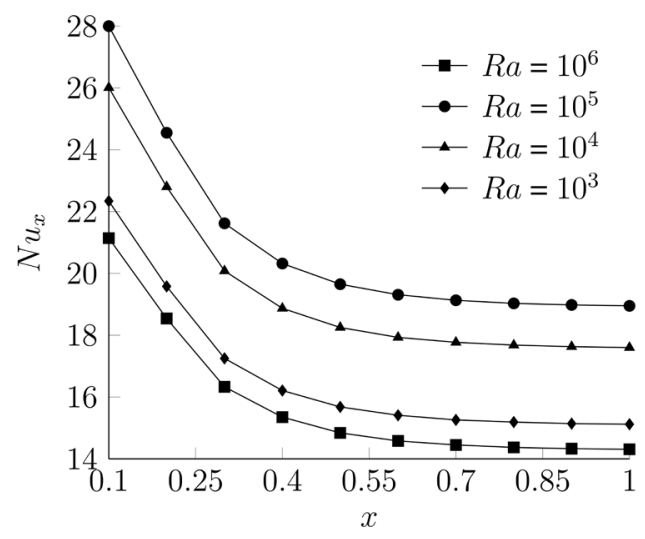

Fig. 7. $N u_{x}$ at $x=0.2 L, B=0.005$ for $\varphi=0.02$

Fig. 8 shows the effect of different $R a$ and $H a$ on the $N u$ at $R e 200$ using $2 \%$ nanofluid. $N u$ decreases with increasing $\mathrm{Ha}$ but not monotonously. The value of $N u$ rises in a weak magnetic range up to $H a 10$. For $\mathrm{Ha}$ above $10, \mathrm{Nu}$ decreases. For a fixed mean velocity of flow, in the perpendicular sectors, the electric 
conduction hindered and the flow velocity accelerated while relatively decelerated in the parallel sectors.

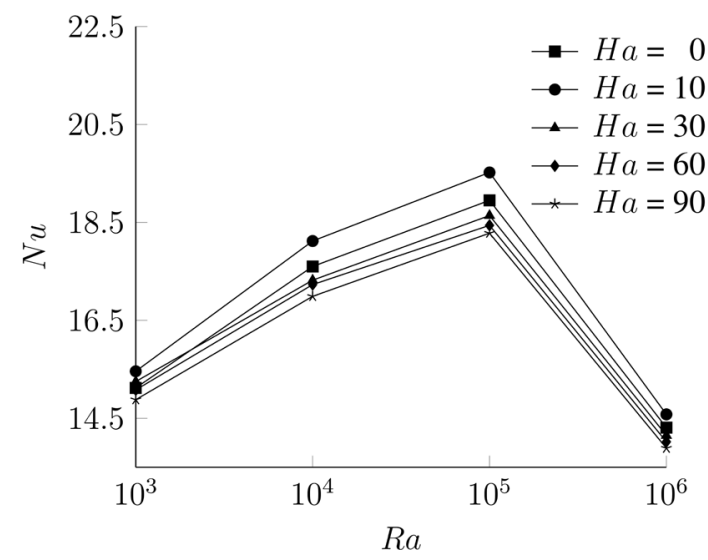

Fig. 8. Effect of $\mathrm{Ha}$ and $\mathrm{Ra}(B=0.005, \varphi=2 \%)$

Therefore, the local mean temperature in the perpendicular sector is lower than the bulk temperature. The electric current pattern established near the edges between the Hartmann walls and the isothermal walls, which combined with the imposed magnetic field, assists the buoyant flow and contrast the viscous forces exerted by the walls. Only for $\mathrm{Ha}$ $>10$ did the decelerating effects of the Lorentz forces near the central regions of the walls become dominant and both peak velocities and $N u$ decreased with $\mathrm{Ha}$. The experimental result of the heat transfer presented the singularity that $N u$ rises in a weak magnetic flux density, though it decreases with increasing magnetic flux density as a whole trend [42]. Tagawa and Ozoe [43] found that a weak magnetic field ( $\mathrm{Ha} 100$ to 200) slightly enhanced heat transfer, causing the average $N u$ to increase by $5 \%$ to $7 \%$, and to enhance the peak velocity attained near the hot and cold walls. Tagawa and Ozoe [44] also confirmed the moderate increase in heat transfer for $\mathrm{Ha} 100$ to 200 in liquid gallium at $\mathrm{Ra}$ 106. $H a=10$ and $R a=10^{5}$ were the best combination with an enhancement up to $3 \% \mathrm{Nu}$.

The critical values for the $R a$ and magnetic field orientation, at which the impacts of the solid volume fraction and magnetic field effects are the most pronounced are reported [30]. The present study is in partial agreement with the finding that when the external magnetic field strength is increased, the average flow drag rises as does the average heat transfer rate [12]. In addition, for smaller nanoparticles, as magnetic field strength intensifies, the peak of the velocity profile near the walls is increased and that at the core region it is decreasing with the enhanced heat transfer rate [10]. The advantage of nanofluids in heat transfer enhancement in the presence of a magnetic field is reported [44]. As the magnetic field strength intensifies, the peak velocity profile near the walls is increased, and the peak velocity profile in the core region is decreased [10].

When the magnetic field is strengthened (higher $\mathrm{Ha}$ ), so does the retarding force, while the peak of the velocity profile in the core region decreases and the velocity profile becomes uniform. The momentum in the core region moves toward the walls, near wall velocity gradients increase, enhancing the slip velocity. The fluid temperature also increases in the fluid due to the additional heating owing to the resistance of fluid flow. These result in an increase in heat transfer rate inside the microtube. The stronger the magnetic field strength, particle-particle and particle-wall interaction will be greater leading to greater clogging in the microtube and greater pressure required to move the fluid through the microtube. The effect is greater closer to the inlet.

However, when the flow strength reaches a certain level ( $R e$ 2500), the pressure needed to move the fluid through the microtube will reach a saturation value with respect to the corresponding value of $\mathrm{Nu}$ (Fig 9). $\mathrm{Nu}$ and the pressure drop increases consecutively with $R e$, with highest $N u$ enhancement of $610 \%$ at $\mathrm{Re} 4000$ using $2 \% \mathrm{Al}_{2} \mathrm{O}_{3}$-water nanofluid in comparison to that at $\operatorname{Re} 200$ using water (Figs. 9 and 10). The application of nanofluids for low $R e$ seems to be highly beneficial in comparison to higher $R e$. The pressure drop shows a similar enhancement in magnitude only. The average $N u$ increases with an increase in $R e$ and nanofluid volume concentration as well as a decrease in nanoparticle size [45] and [46]. The enhancement of heat transfer is reported to be around $22 \%$ using the $1 \% \mathrm{Al}_{2} \mathrm{O}_{3}$-water nanofluid compared to the water and has a higher friction factor than water does [47].

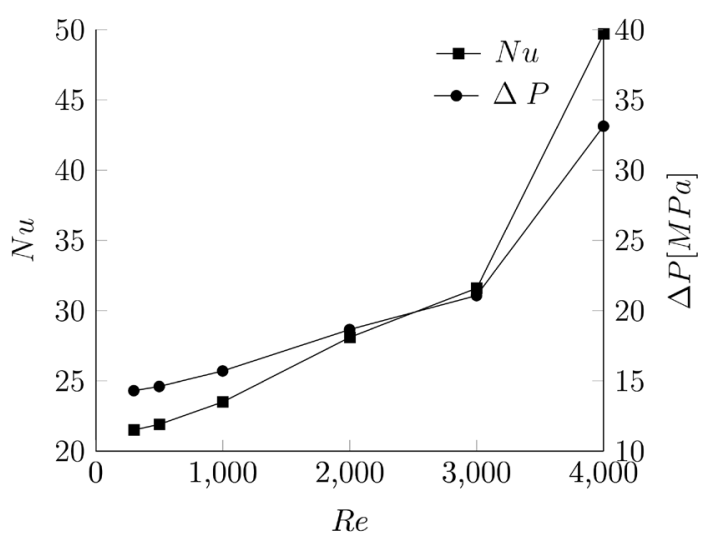

Fig. 9. Effect of Re on $N u$ and pressure drop 


\section{VALIDATION AND GRID INDEPENDENCE}

We have conducted mesh testing to ensure grid independence. Calculations of different mesh cases were carried out in $2 \% \mathrm{Al}_{2} \mathrm{O}_{3}$-water nanofluid of $R a=10^{6}, B=0.005$ and $R e=50$. Having studied grid independence to evaluate the heat transfer of nanofluid in the microtube in terms of the average $\mathrm{Nu}$ as shown in Table 1, the grid independence was ensured, and a lattice with $800 \times 14$ nodes found appropriate for the next computations.

Table 1. Results of grid independence test

\begin{tabular}{cccc}
\hline $\begin{array}{c}\text { Grids } \\
(x \text { direction })\end{array}$ & $\begin{array}{c}\text { Grids } \\
(y \text { direction })\end{array}$ & $\begin{array}{c}\text { Grid } \\
\text { nodes }\end{array}$ & $\begin{array}{c}\text { Average } \\
\text { Nusselt No. }\end{array}$ \\
\hline 700 & 12 & 8400 & 16.68662 \\
\hline 900 & 10 & 9000 & 16.11631 \\
\hline 800 & 12 & 9600 & 15.45408 \\
\hline 700 & 10 & 7000 & 14.99470 \\
\hline 700 & 14 & 9800 & 13.96442 \\
\hline 900 & 14 & 12600 & 13.95444 \\
\hline 800 & 14 & 11200 & 13.95020 \\
\hline 800 & 16 & 12800 & 12.14082 \\
\hline 800 & 18 & 14400 & 11.46672 \\
\hline 900 & 18 & 16200 & 10.55592 \\
\hline
\end{tabular}

The dependency of the grid on a microtube is performed and shows an excellent agreement between the $N u$ results in the $R e$ range relevant for the present study (200 to 4000). The $y^{+}$values are much smaller than $1(0.054,0.063$, and 0.051 , respectively). For a laminar flow, the results match very well with each other, under the condition that $y^{+} \leq 1$ recommended for high accuracy. For the $900 \times 14$ mesh, $y^{+}>1$ is observed for $R e$ around 50000. Around this Reynolds number, the $N u$ starts diverging from the expected solution and leads to increasingly wrong results, strongly underestimating $\mathrm{Nu}$. The same effect appears for the $800 \times 14$ mesh for $R e>41000$. The $800 \times 16$ mesh shows good agreement with the $N u$ results until $\operatorname{Re}>52000$.

For further validation, we have compared the proposed LBM for incompressible fluid simulation results with the experimental results of [48]. Fig. 10 shows that the comparisons are in excellent agreement with the maximum deviation of less than $6 \%$.

Fig. 11 shows the dimensionless velocity profiles, at different $\varphi=0$ to 0.04 along the microtube wall at $x=0.2 \mathrm{~L}$ for $B=0.005$ and $y=0$ to 1 . The fully developed velocity condition is observed after a short entrance length of $x=0.03 \mathrm{~L}$. The slip coefficient leads to generate the slip velocity at $Y=0$ and $Y=$
1 which is well obvious in Fig. 11. However, it has the maximum value at the entrance and then decreases along the microtube. In contrary of usual macro scale flows in tubes, the maximum value of velocity (1.48) is less than 1.5 in a fully developed region due to slip velocity on the walls. It can be seen from the figure that with the increase in volume fraction, the velocity curves become closer to the $y$-axis. The velocity curve becomes shrunk compared with the pure fluid. It is observed that the increase of solid concentration leads to enhanced effective thermal conductivity and the decrease of velocity.

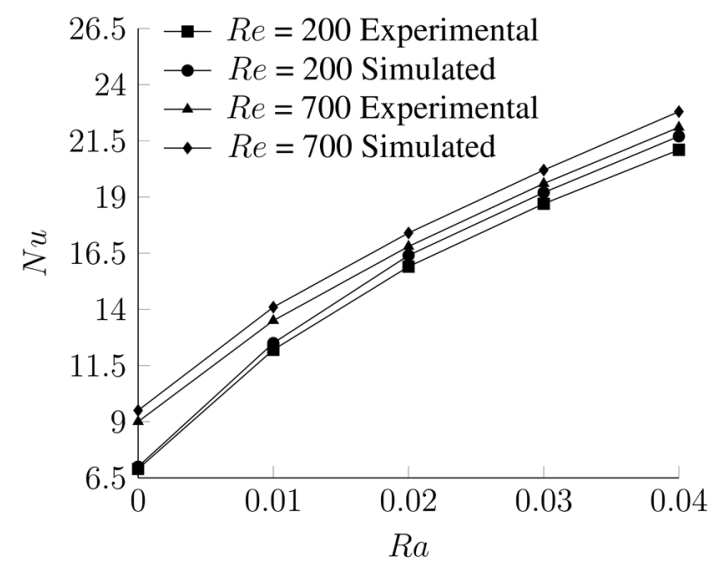

Fig. 10. Comparison of simulated and experimental data [48]

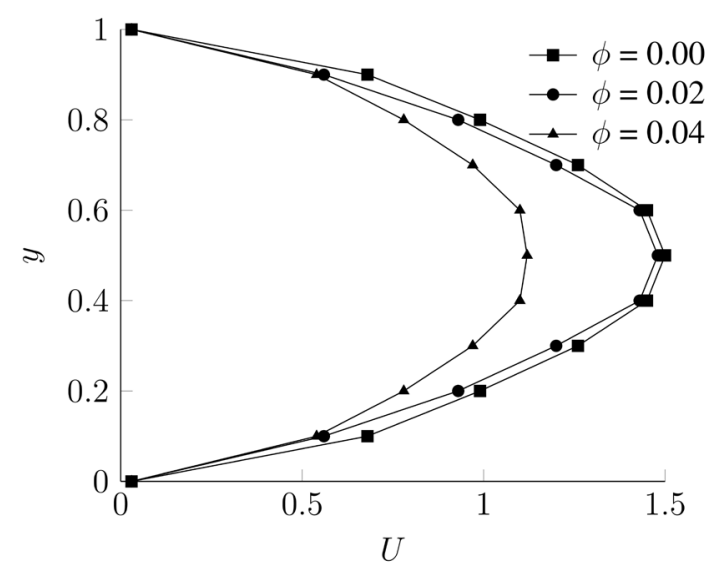

Fig. 11. Velocity profiles at $\varphi=0$ to 0.04

Fig. 12 shows the dimensionless temperature profiles along the microtube wall at $x=0.2 \mathrm{~L}$ for $B=$ 0.005 and $\varphi=0.02$ as a function of $y$. The minimum value of nanofluid temperature (1.99) is less than 2 in the fully developed region due to temperature jump. Fig. 12 represents the temperature jump generated. However, there is a very small value of the temperature jump in the fully developed region. 


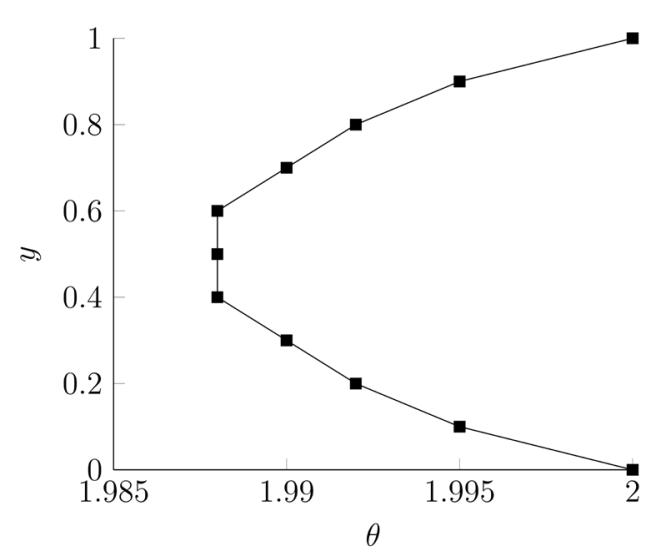

Fig. 12. Temperature profiles $(x=0.2 \mathrm{~L}, B=0.005, \varphi=0.02)$

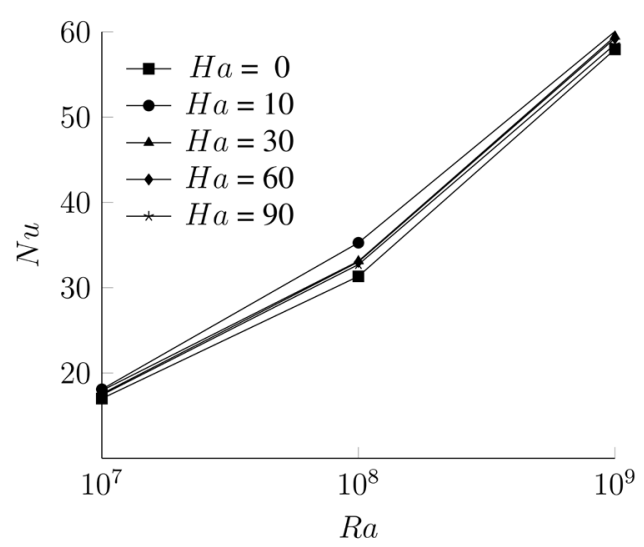

Fig. 13. Effect of $\mathrm{Ha}$ and $\mathrm{Ra}$ on $\mathrm{Nu}(B=0.005, \varphi=2 \%)$ for turbulent flow

Fig. 13 shows the average $N u$ for different $R a$ and $\mathrm{Ha}$ for turbulent flow. The trend of enhancement of average $\mathrm{Nu}$ with the $\mathrm{Ha}$ is not the same at various $R a$. Heat transfer augmentation takes place at $\mathrm{Ra} 10^{7}$ to $10^{9}$ by $H a 0$ to 10 , while it commences dropping from $\mathrm{Ha} 30$ to 90 . Similar heat transfer enhancement of a magnetic field at $\mathrm{Ha} 25$ and retarding effect from $H a 25$ to 100 at the $R a 10^{7}$ and $10^{9}$ in a square cavity was reported [49]. For $H a=0$ and 10, the isotherms pattern is like a plume, indicating strong convection as expected, since $R a$ is higher than $10^{5}$. In the absence of a magnetic field $(H a=0)$, the isoconcentration curves are symmetric and almost uniformly distributed in the entire enclosure. However, the isoconcentration curves become asymmetric in the presence of a magnetic field. An increased Hartmann number, i.e. a stronger magnetic field, diminishes the flow strength within the cavity. The magnetic susceptibility due to the applied magnetic field over a moving fluid creates a Lorentz force that has the tendency to oppose the flow; consequently, streamlines weaken inside the cavity. Thus, when $\mathrm{Ha}$ is increased to 30 , it is found that the vortices are no more symmetric. This occurs due to the presence of magnetic field, which gives rise to the Lorentz force and tries to retard the convection. When $\mathrm{Ha}$ is increased to 60 and 90 , isotherms are almost parallel that is Hartmann number acts against convection inside the cavity. Increasing $\mathrm{Ha}$ further $(H a=90)$ reduces the strength of the vortex, and the eyes of the vortices are shifted to the bottom region of the cavity.

\section{CONCLUSIONS}

The present paper investigates the magnetic field applied to forced convection laminar and turbulent heat transfer in a microtube filled with $\mathrm{Al}_{2} \mathrm{O}_{3}$-water nanofluid. This has been simulated by MATLAB based LBM-BGK method. The effects of $R e, R a$, nanoparticle volume fraction, $\mathrm{Ha}$, slip coefficient and axial distance on the characteristics of flow and heat transfer have been examined. The major findings of this paper are:

- The $N u$ and pressure drop increase with the Re. However, the application of nanofluids for low $R e$ seems to be highly beneficial in comparison to higher $R e$.

- The increase of volume fraction of $\mathrm{Al}_{2} \mathrm{O}_{3}$ nanoparticles in water enhances the heat transfer in the microtube at various $R a$. However, there is a critical $R a$ for heat transfer enhancement while using nanofluids, beyond which the enhancement rate will be reduced. The heat transfer of nanofluid increases with the increase of $\mathrm{Ha}$ for a fixed $\mathrm{Ra}$ and nanoparticle volume fraction up to a lower $\mathrm{Ha}$ and thereafter the heat transfer decreases. A critical lower $R a$ pronounces the effect of $H a$.

- The larger value of slip coefficient corresponds to a higher temperature jump and lower heat transfer. Nanofluid with $2 \%$ volume fraction at low values of slip coefficient can increase heat transfer in a microtube. However, the effect of volume fractions more pronounced compared to slip coefficient.

- The impact of nanoparticle volume fraction on $N u$ is small when $10^{4<R a}>10^{5}$ and negligible at all other $R a$ values of the investigation.

- Heat transfer augmentation takes place with turbulent flow at all $\mathrm{Ra}$ by $\mathrm{Ha}$ up to 10 while it drops from $\mathrm{Ha}$ above 30.

- Decreasing the axial distance enhances the Nusselt number. 


\section{NOMENCLATURES}

$c \quad \Delta x / \Delta t$, Microscopic velocity vector, $\left[\mathrm{ms}^{-1}\right]$

$D_{H}=2 h$ Hydraulic diameter, $[\mathrm{m}]$

$e \quad$ Internal energy density, $\left[\mathrm{J} \mathrm{m}^{-3}\right]$

$F \quad$ External force term, $[\mathrm{N}]$

$h \quad$ Height of microtube, $[\mathrm{m}]$

$k \quad$ Thermal conductivity coefficient, $\left[\mathrm{Wm}^{-1} \mathrm{~K}^{-1}\right]$

Pr Prandtl number, [-]

$\alpha, \chi \quad$ Thermal diffusivity, $\left[\mathrm{m}^{2} \mathrm{~s}^{-1}\right]$

$\beta \quad$ Slip/thermal expansion coefficient, $\left[\mathrm{K}^{-1}\right]$

$v \quad$ Kinematic viscosity, $\left[\mathrm{m}^{2} \mathrm{~s}^{-1}\right]$

$\rho \quad$ Density, $\left[\mathrm{kg} \mathrm{m}^{-3}\right]$

$\Delta P \quad$ Pressure drop, $[\mathrm{Pa}]$

$\Delta x \quad$ lattice spacing, [-]

$\Delta T \quad$ Temperature difference, $[\mathrm{K}]$

$e \quad$ Equilibrium

$f \quad$ Base fluid

$i \quad$ Inlet flow, lattice directions

$\alpha \quad x-y$ geometry components

$\delta_{u} \quad$ lattice step $u,[-]$

$\delta_{t} \quad$ Time step $t,[-]$

$\omega_{i} \quad$ Weighting factor, [-]

\section{REFERENCES}

[1] Karimipour, A., Esfe, M.H., Safaei, M.R., Semiromi, D.T., Jafari, S., Kazi, S.N. (2014). Mixed convection of copper-water nanofluid in a shallow inclined lid driven cavity using the lattice Boltzmann method. Physica A: Statistical Mechanics and its Applications, vol. 402, p. 150-168, D0l:10.1016/j. physa.2014.01.057.

[2] Bompos, D.A., Nikolakopoulos, P.G. (2016). Experimental and analytical investigations of dynamic characteristics of magnetorheological and nanomagnetorheological fluid film journal bearing. Journal of Vibration and Acoustics, vol. 138, no. 3, p. 1-7, Dol:10.1115/1.4032900.

[3] Nkurikiyimfura, I., Wang, Y., Pan, Z. (2013). Heat transfer enhancement by magnetic nanofluids - A review. Renewable and Sustainable Energy Reviews, vol. 21, p. 548-561, DOI:10.1016/j.rser.2012.12.039.

[4] Samouhos, S., McKinley, G. (2006). Carbon nanotubemagnetite composites, with applications to developing unique magnetorheological fluids. Journal of Fluids Engineering, vol. 129, no. 4, p. 429-437, Dol:10.1115/1.2436581.

[5] Nakatsuka, K., Jeyadevan, B., Neveu, S., Koganezawa, H. (2002). The magnetic fluid for heat transfer applications. Journal of Magnetism and Magnetic Materials, vol. 252, p. 360-362, DOI:10.1016/S0304-8853(02)00683-2.

[6] Mokeev, A.A., Gubarev, S.A., Korobko, E.V., Bedik, N.A. (2013). Microconvection heat transfer in electrorheological fluids in rotating electric field. Journal of Physics: Conference Series, vol. 412, conf. 1, 13 th International Conference on Electrorheological Fluids and Magnetorheological Suspensions, p. 1-13, D0I:10.1088/17426596/412/1/012008.
[7] Rahim, M.S.A., Ismail, I. (2015). Review of magnetorheological fluids and nanofluids thermal behaviour. The 3rd International Conference on Mechanical Engineering Research, Pahang, p. 1-10, DOI:10.1088/1757-899X/100/1/012040.

[8] Shah, K., Choi, S.B., Choi, H.J. (2015). Thermorheological properties of nano magnetorheological fluid in dynamic mode: experimental investigation. Smart Materials and Structures, vol. 24, no. 5, DOl:10.1088/0964-1726/24/5/057001.

[9] Begum, R., Basit, M.A. (2008). Lattice Boltzmann Method and its Applications to Fluid Flow Problems. European Journal of Scientific Research, vol. 22, no. 2, p. 216-231.

[10] Malvandi, A., Ganji, D.D. (2014). Magnetohydrodynamic mixed convective flow of Al203-water nanofluid inside a vertical microtube. Journal of Magnetism and Magnetic Materials, vol. 369, p. 132-141, D0I:10.1016/j.jmmm.2014.06.037.

[11] Niu, J., Fu, C., Tan, W. (2012). Slip-flow and heat transfer of non-Newtonian nanofluid in a microtube. PLOS ONE, vol. 7, no. 5, p. 1-9, Dol:10.1371/journal.pone.0037274.

[12] Lo, K.J., Weng, H.C. (2015). Convective heat transfer of magnetic nanofluids in a microtube. Smart Science, vol. 3, no. 2, p. 56-64, DOI:10.1080/23080477.2015.11665637.

[13] Goodarzi, M., Safaei, M.R., Karimipour, A., Hooman, K., Dahari, M., Kazi, S.N., Sadeghinezhad, E. (2014). Comparison of the finite volume and lattice Boltzmann methods for solving natural convection heat transfer problems inside cavities and enclosures. Abstract and Applied Analysis, vol. 2014, p. 1-15, DOl:10.1155/2014/762184.

[14] Cetin, B., Zeinali, S. (2014). Analysis of heat transfer and entropy generation for a low-Peclet-number microtube flow using a second-order slip model: an extended-Graetz problem. Journal of Engineering Mathematics, vol. 89, no. 1, p. 13-25, DOI:10.1007/s10665-014-9704-7.

[15] Tang, K., Huang, J.L., Jin, T., Wu, M.X. (2015). An experimentbased comparative investigation of correlations for microtube gas flow. Sadhana, vol. 40, no. 2, p. 537-547, Dol:10.1007/ s12046-014-0326-6.

[16] He, X., Chen, S., Doolen, G.D. (1998). A novel thermal model for the lattice Boltzmann method in incompressible limit. Journal of Computational Physics, vol. 146, no. 1, p. 282-300, D0l:10.1006/jcph.1998.6057.

[17] D'Orazio, A., Corcione, M., Celata, G.P. (2004). Application to natural convection enclosed flows of a lattice Boltzmann BGK model coupled with a general-purpose thermal boundary condition. International Journal of Thermal Sciences, vol. 43, no. 6, p. 575-586, Dol:10.1016/j.jithermalsci.2003.11.002.

[18] Raisi, A., Ghasemi, B., Aminossadati, S.M. (2011). A numerical study on the forced convection of laminar nanofluid in a microchannel with both slip and no-slip conditions. Numerical Heat Transfer, Part A: Applications, vol. 59, no. 2, p. 114-129, DOI:10.1080/10407782.2011.540964.

[19] Guo, Y., Qin, D., Shen, S., Bennacer, R. (2012). Nanofluid multiphase convective heat transfer in closed domain: Simulation with lattice Boltzmann method. International Communications in Heat and Mass Transfer, vol. 39, no. 3, p. 350-354, DOI:10.1016/j.icheatmasstransfer.2011.12.013.

[20] Ay, C., Young, C.-W., Young, C.-F. (2012). Application of lattice Boltzmann method to the fluid analysis in a rectangular micro 
channel. Computers \& Mathematics with Applications, vol. 64, no. 5, p. 1065-1083, D0l:10.1016/j.camwa.2012.03.025.

[21] Yang, Y.-T., Lai, F.-H. (2011). Numerical study of flow and heat transfer characteristics of alumina-water nanofluids in a microchannel using the lattice Boltzmann method. International Communications in Heat and Mass Transfer, vol. 38, no. 5, p. 607-614, D0l:10.1016/j. icheatmasstransfer.2011.03.010.

[22] Takabi, B., Shokouhmand, H. (2015). Effects of Al203-Cu/ water hybrid nanofluid on heat transfer and flow characteristics in turbulent regime. International Journal of Modern Physics C, vol. 26, no. 4, DOl:10.1142/S0129183115500473.

[23] Kakaç, S., Pramuanjaroenkij, A. (2016). Analysis of convective heat transfer enhancement by nanofluids: single-phase and two-phase treatments. Journal of Engineering Physics and Thermophysics, vol. 89, no. 3, p. 758-793, D0l:10.1007/ s10891-016-1437-1.

[24] Takabi, B., Salehi, S. (2014). Augmentation of the heat transfer performance of a sinusoidal corrugated enclosure by employing hybrid nanofluid. Advances in Mechanical Engineering, vol. 6, Dol:10.1155/2014/147059.

[25] Chapman, S., Cowling, T.G. (1999). The Mathematical Theory of Non-uniform Gases. 3rd ed., Cambridge University Press, Cambridge.

[26] Sajjadi, A.H., Kefayati, R. (2015). Lattice Boltzmann simulation of turbulent natural convection in tall enclosures. Thermal Science, vol. 19, no. 1, p. 155-166, D0l:10.2298/ tsci120105066s.

[27] Alamyane, A.A., Mohamad, A.A. (2010). Simulation of forced convection in a channel with extended surfaces by the lattice Boltzmann method. Computers \& Mathematics with Applications, vol. 59, no. 7, p. 2421-2430, D0I:10.1016/j. camwa.2009.08.070.

[28] Arya, G., Chang, H.-C., Maginn, E.J. (2003). Molecular simulations of Knudsen wall-slip: effect of wall morphology. Molecular Simulation, vol. 29, no. 10-11, p. 697-709, DOl:10.1080/0892702031000103257.

[29] Dellar, P.J. (2002). Lattice kinetic schemes for magnetohydrodynamics. Journal of Computational Physics, vol. 179, no. 1, p. 95-126, Dol:10.1006/jcph.2002.7044.

[30] Succi, S. (2002). Mesoscopic modeling of slip motion at fluidsolid interfaces with heterogeneous catalysis. Physical Review Letters, vol. 89, no. 6, p. 064502-1-064502-4, D0l:10.1103/ PhysRevLett.89.064502.

[31] Xing, Y., Zhi, T., Haiwang, L., Yitu, T. (2016). Experimental investigation of surface roughness effects on flow behavior and heat transfer characteristics for circular microchannels. Chinese Journal of Aeronautics, vol. 29, no. 6, p. 1575-1581, D0I:10.1016/j.cja.2016.10.006.

[32] Zhang, C., Chen, Y., Deng, Z., Shi, M. (2012). Role of rough surface topography on gas slip flow in microchannels. Physical Review E, vol. 86, no. 1, p. 016319, D0l:10.1103/ PhysRevE.86.016319.

[33] Aminossadati, S.M., Raisi, A., Ghasemi, B. (2011). Effects of magnetic field on nanofluid forced convection in a partially heated microchannel. International Journal of Non-Linear Mechanics, vol. 46, no. 10, p. 1373-1382, D0I:10.1016/j. ijnonlinmec.2011.07.013.
[34] Kalteh, M., Abbassi, A., Saffar-Avval, M., Harting, J. (2011). Eulerian-Eulerian two phase numerical simulation of nanofluid laminar forced convection in a microchannel. International Journal of Heat and Fluid Flow, vol. 32, no. 1, p. 107-116, D0l:10.1016/j.ijheatfluidflow.2010.08.001.

[35] Karimipour, A., Nezhad, A.H., D'Orazio, A., Esfe, M.H., Safaei, M.R., Shirani, E. (2015). Simulation of copper-water nanofluid in a microchannel in slip flow regime using the lattice Boltzmann method. European Journal of Mechanics - B/Fluids, vol. 49, p. 89-99, D0l:10.1016/j.euromechflu.2014.08.004.

[36] Ahmed, M., Eslamian, M. (2015). Laminar forced convection of a nanofluid in a microchannel: effect of flow inertia and external forces on heat transfer and fluid flow characteristics. Applied Thermal Engineering, vol. 78, p. 326-338, DOI:10.1016/j.euromechflu.2014.08.004.

[37] Yang, C., Li, W., Sano, Y., Mochizuki, M., Nakayama, A. (2013). On the anomalous convective heat transfer enhancement in nanofluids: a theoretical answer to the nanofluids controversy. Journal of Heat Transfer, vol. 135, no. 5, p. 545041-545049, DOI:10.1115/1.4023539.

[38] Zhou, W., Yan, Y. (2014). Lattice Boltzmann simulation of magnetic field effects on nanofluid. $4^{\text {th }}$ Micro and Nano Flows Conference. University College London, London.

[39] Taher, M.A., Lee, Y.W., Kim, H.D. (2013). Heat transfer enhancement of $\mathrm{Cu}-\mathrm{H} 2 \mathrm{O}$ nanofluid with internal heat generation using LBM. Open Journal of Fluid Dynamics, vol. 3 , p. 92-99, D0l:10.4236/ojfd.2013.32A015.

[40] Mliki, B., Abbassi, M.A., Guedri, K., Omri, A. (2015). Lattice Boltzmann simulation of natural convection in an L-shaped enclosure in the presence of nanofluid. Engineering Science and Technology, an International Journal, vol. 18, no. 3, p. 503-511, D0I:10.1016/J.jestch.2015.04.008.

[41] He, Y., Qi, C., Hu, Y., Qin, B., Li, F., Ding, Y. (2011). Lattice Boltzmann simulation of alumina-water nanofluid in a square cavity. Nanoscale Research Letters, vol. 6, p. 184-191, DOI:10.1186/1556-276X-6-184.

[42] Miyazaki, K., Inouet, H., Kimotott, T., Yamashita, S., Inoue, S., Yamaoka, N. (1986). Heat transfer and temperature fluctuation of lithium flowing under transverse magnetic field. Journal of Nuclear Science and Technology, vol. 23, no. 7, p. 582-593, DOI:10.1080/18811248.1986.9735026.

[43] Tagawa, T., Ozoe, H. (1997). Enhancement of heat transfer rate by application of a static magnetic field during natural convection of liquid metal in a cube. Journal of Heat Transfer, vol. 119, no. 2, p. 265-271, Dol:10.1115/1.2824219.

[44] Tagawa, T., Ozoe, H. (1998). Enhanced heat transfer rate measured for natural convection in liquid gallium in a cubical enclosure under a static magnetic field. Journal of Heat Transfer, vol. 120, no. 4, p. 1027-1032, DOI:10.1115/1.2825886.

[45] Malvandi, A., Safaei, M.R., Kaffash, M.H., Ganji, D.D. (2015). MHD mixed convection in a vertical annulus filled with Al203water Nanofluid considering nanoparticle migration. Journal of Magnetism and Magnetic Materials, vol. 382, p. 296-306, D0l:10.1016/J.jmmm.2015.01.060.

[46] Kalteh, M., Abbassi, A., Saffar-Avval, M., Frijns, A., Darhuber, A. Harting, J. (2012). Experimental and numerical investigation of nanofluid forced convection inside a wide microchannel 
heat sink. Applied Thermal Engineering, vol. 36, p. 260-268, DOI:10.1016/J.applthermaleng.2011.10.023.

[47] Salman, B.H., Mohammed, H.A., Munisamy, K.M., Kherbeet, A.S. (2014). Three-dimensional numerical investigation of nanofluids flow in microtube with different values of heat flux. Heat Transfer-Asian Research, vol. 44, no. 7, p. 599-619, DOI:10.1002/htj.21139.

[48] Salman, B.H., Mohammed, H.A., Kherbeet, A.S. (2014). Numerical and experimental investigation of heat transfer enhancement in a microtube using nanofluids. International Communications in Heat and Mass Transfer, vol. 59, p. 88100, D0I:10.1016/j.icheatmasstransfer.2014.10.017.

[49] Sajjadi, H., Kefayati, G.H.R. (2014). MHD turbulent and laminar natural convection in a square cavity utilizing lattice Boltzmann method. Heat Transfer-Asian Research, vol. 45, no. 8, p. 795-814, D0l:10.1002/htj.21191. 